\title{
ResearchOnline@JCU
}

This is the author-created version of the following work:

Haig, Jordahna, Ascough, Philippa, Wurster, Christopher M., and Bird, Michael I. (2020) A rapid throughput technique to isolate pyrogenic carbon by hydrogen pyrolysis for stable isotope and radiocarbon analysis. Rapid Communications in Mass Spectrometry, 34 (10) .

Access to this file is available from:

https://researchonline.jcu.edu.au/67133/

(C) 2020 John Wiley \& Sons, Ltd.

Please refer to the original source for the final version of this work: 


\section{A rapid throughput technique to isolate pyrogenic carbon by hydrogen pyrolysis for}

\section{2 stable isotope and radiocarbon analysis}

3 Jordahna Haig ${ }^{1}$, Philippa L. Ascough ${ }^{2}$, Christopher M. Wurster ${ }^{1}$, Michael I. Bird ${ }^{1}$

$4{ }^{1}$ College of Science and Engineering, ARC Centre of Excellence of Australian Biodiversity 5 and Heritage and Centre for Tropical Environmental and Sustainability Science, James Cook 6 University, Cairns, QLD 4870, Australia

$7 \quad{ }^{2}$ NERC Radiocarbon Facility, SUERC, Scottish Enterprise Technology Park, Rankine 8 Avenue, East Kilbride, G75 0QF, UK

9

Keywords: Radiocarbon pretreatment; pyrogenic carbon; hydrogen pyrolysis; charcoal

\section{Abstract}

RATIONALE: Rapid, reliable isolation of Pyrogenic Carbon (PyC; char, soot, black carbon; biochar) for determination of stable carbon isotope $\left(\delta^{13} \mathrm{C}\right)$ composition and radiocarbon $\left({ }^{14} \mathrm{C}\right)$ dating is needed across multiple fields of research in geoscience, environmental science and archaeology. Many current techniques do not provide reliable isolation from contaminating organics and/or are relatively time consuming to employ. Hydrogen pyrolysis (HyPy) does provide reliable isolation of $\mathrm{PyC}$ but the current methodology is time consuming.

METHODS: We explored the potential for subjecting multiple samples to HyPy analysis by placing up to nine individual samples in custom designed borosilicate sample vessels in a single reactor run. We tested for cross contamination between samples in the same run using materials with highly divergent radiocarbon activities ( $\sim 0.04$ to $116.3 \mathrm{pMC}), \delta^{13} \mathrm{C}$ values (- 
2311.9 to $-26.5 \%$ ) and labile carbon content. We determined ${ }^{14} \mathrm{C} /{ }^{13} \mathrm{C}$ by accelerator mass

24 spectrometry and $\delta^{13} \mathrm{C}$ values by elemental analyser coupled to continuous flow isotope ratio 25 mass spectrometer.

26 RESULTS: Very small but measurable transfer between samples of highly divergent isotope 27 composition was detectable. Where samples are of broadly similar composition, this cross 28 contamination is considered negligible with respect to measurement uncertainty. Where 29 samples are of divergent composition, it was found that placing a sample vessel loaded with 30 silica mesh adsorbent between samples eliminated measurable cross-contamination in all 31 cases for both ${ }^{14} \mathrm{C} /{ }^{13} \mathrm{C}$ and $\delta^{13} \mathrm{C}$ values.

32 CONCLUSION: It is possible to subject up to seven samples to HyPy in the same reactor 33 run for determination of radiocarbon content and $\delta^{13} \mathrm{C}$ value without diminishing the 34 precision or accuracy of the results. This approach enables an increase in sample throughput 35 of 300-600\%. HyPy process background values are consistently lower than the nominal 36 laboratory process background for quartz tube combustion in the NERC Radiocarbon 37 Laboratory, indicating that HyPy may also be advantageous as a relatively 'clean' 38 radiocarbon pretreatment method.

\section{Introduction}

Pyrogenic carbon (PyC, also known as char, black carbon, biochar) is derived from the 41 incomplete combustion (pyrolysis) of organic matter during natural fires, the purposeful 42 pyrolysis of biomass to create 'biochar', or via fossil fuel combustion [1,2]. PyC is a ubiquitous component of carbon in soils, sediments, atmospheric particulates, fresh and marine waters, in both dissolved and/or particulate (microscopic and macroscopic) forms. 
$\mathrm{PyC}$ is important as a poorly understood, slow-cycling component of the global carbon cycle, that is now known to comprise a significant, but as yet unquantified, pool that contains some of the most recalcitrant organic carbon on Earth $[3,4]$. In the form of biochar, PyC also has the potential to sequester significant amounts of carbon over time periods far exceeding that of biomass, therefore offsetting at least a proportion of current anthropogenic $\mathrm{CO}_{2}$ emissions [5]. PyC is also useful as a form of recalcitrant carbon that can provide a valuable palaeoenvironmental proxy through its stable isotope composition [6]. It is one of the most common materials (as 'charcoal') used for construction of radiocarbon chronologies in support of archaeological and Quaternary environmental studies.

Quantification and isolation of $\mathrm{PyC}$ has long proven problematic. This is because PyC represents a continuum of complex molecular components with differing chemical and spectroscopic properties. Each method targets a particular 'window' along this continuum, meaning the 'PyC' isolated by two differing methods can be chemically quite distinct. Thus, while many techniques have been developed to analyse $\mathrm{PyC}$ in a range of environmental matrices, these techniques produce widely divergent results for the same samples, the same technique has produced divergent results across different laboratories and some techniques are not broadly applicable across the range of matrices that can contain PyC [7]. The key difficulty however, is that many methods rely on an operational definition of $\mathrm{PyC}$ in order to achieve isolation. This often requires that what remains after the process must, by definition, be $\mathrm{PyC}$, and that quantification relies upon a single parameter, such as weight loss during processing. Problems arise if non-PyC material is not removed e.g. due to hydrophobic protection of plant waxes in aqueous solutions [8], or if $\mathrm{PyC}$ is formed de novo during oxidative reactions [9]. Procedures isolating $\mathrm{PyC}$ on a secure chemically-defined basis, are therefore preferable. 
Hydrogen pyrolysis (HyPy) was originally used as a method for the efficient conversion of macromolecular organic matter to dichloromethane soluble oils with conversions near $100 \%$ possible using high hydrogen pressures (>10 MPa) at high temperature [10]. During these experiments, it was found that in some cases substantial amounts of highly aromatic macromolecular carbon remained in the residues, attributed to PyC [11].

The possibility that HyPy could be used to quantify PyC was first discussed by Ascough et al $[12,13]$, who developed a hydrogen pyrolysis procedure specific to eliminating labile components and leaving only polyaromatic carbon in the residue. Meredith et al [14] demonstrated that HyPy yielded results within the range of other techniques for $\mathrm{PyC}$ in a range of matrices using the black carbon ring trial samples of Hammes et al [7]. Meredith et al [14] also demonstrated that HyPy was able to remove potentially interfering non-pyrogenic materials with the exception of anthracite, and that the component isolated by HyPy is chemically highly consistent, being polyaromatic carbon with a ring size greater than 7 (coronene). Smaller pyrogenic polyaromatic molecules can be collected by cryogenic trapping downstream of the HyPy reactor for separate quantification if required [15-17]. More recent work has demonstrated the utility of HyPy analysis across a range of environmental matrices, for simple quantification [18-20] with good reproducibility within and between laboratories [21], as well for stable isotope analysis [6,22,23] and radiocarbon dating $[12,13,24,25]$.

While the advantages of HyPy for the isolation and quantification of a well-defined component of PyC have now been clearly demonstrated, the technique remains relatively slow. The instrument reactor conventionally accommodates one sample at a time and a single run takes approximately 43 minutes. While the technique is not more time consuming than many other competing techniques, the current throughput limits the scale of projects that can be undertaken. At the same time, research interest in PyC is growing rapidly, particularly for 
94 carbon-cycle science applications. For example, measuring $\mathrm{PyC}$ abundance and turnover

95 times by ${ }^{14} \mathrm{C}$, which by their nature require high-volume throughput. The general need to improve throughput is now driving modifications to other established techniques such as

97 BPCA analysis [26].

Here we report on the results of experiments designed to test whether multiple samples can be run simultaneously in a single HyPy reactor for accelerated throughput and efficiency, 100 without either cross contaminating the samples or degrading the accuracy or precision of 101 individual stable isotope and radiocarbon analyses.

\section{Materials and Methods}

\subsection{Samples}

The samples for this study were chosen to provide the largest possible contrast in lability of material (i.e. degree of aromaticity), and in carbon isotope $\left(\delta^{13} \mathrm{C}\right.$ and $\left.{ }^{14} \mathrm{C}\right)$ composition. Five matrixes were chosen for experiments designed to test the degree to which combining multiple samples with divergent characteristics in a single reactor run effected the measured PyC abundance, $\delta{ }^{13} \mathrm{C}$ and ${ }^{14} \mathrm{C}$ measurements of individual samples in the same reactor (see Table 1.). Throughout this manuscript, error is reported as $2 \sigma$.

Three materials of known radiocarbon content were chosen for experiments designed to test, 1) the ${ }^{14} \mathrm{C}$ background of the HyPy process itself, and 2) the degree of inter-sample

112 transfer of carbon when combining multiple samples with different ${ }^{14} \mathrm{C}$ content in a single 113 HyPy run:

(i) TIRI barley mash (TBM): This standard originates from the Third International

115 Radiocarbon Intercomparison [27]. It is composed of lignocellulosic biomass, known to be entirely labile during HyPy. The consensus value for TBM is $116.35 \pm 0.016$ pMC. TBM was

117 used as a source of excess labile $\mathrm{C}$ from a sample in $\mathrm{HyPy}$, which thus had the potential to be 
118 transferred to other samples in the same HyPy reactor. The relatively high ${ }^{14} \mathrm{C}$ content of

119 TBM makes it possible to detect even small quantities of cross-contamination, when used with a contrasting RCD (radiocarbon dead) material (below).

(ii) Anthracite: This is a NERC Radiocarbon Facility in-house process standard anthracite [28]. It is a highly aromatic material, and anthracite coal is known to be resistant to HyPy [14]. This material is 'radiocarbon-dead', being much greater than 50,000 years in age, and is in use as a process background material for radiocarbon analyses. For the quartz tube combustion method of $\mathrm{CO}_{2}$ production (the process applied in this study), the long-term average background value at the NERC Radiocarbon laboratory is $0.17 \pm 0.02 \mathrm{pMC}$. The anthracite standard was used to identify whether the HyPy ${ }^{14} \mathrm{C}$ background was commensurate with this value before, during, and after the experiments described below, and to provide confidence that any observed variation in ${ }^{14} \mathrm{C}$ during multiple simultaneous HyPy runs can be ascribed to a specific process (i.e. transfer of carbon between sample vessels within the reactor for this specific experiment), and not an inherent feature of the method itself.

(iii) $R D C$ : This is a radiocarbon-dead charcoal produced experimentally from a log of 134 Miocene age and previously characterized by Bird et al [29]. It is a material resistant to conversion during HyPy, with a radiocarbon activity equivalent to $0.04 \pm 0.02 \mathrm{pMC}$. This material is also highly aromatic, similar to the anthracite coal standard, but was included as it represents a more typical form of natural PyC (i.e. the product of biomass that has been thermally altered during fire). During the experiments below, RDC was used to represent material into which the introduction of cross-contamination (from TBM) during HyPy could 140 be monitored. 
Hydrogen pyrolysis has been described in detail in a number of publications $[10,30,12,14]$.

143 Briefly, 25-100 mg aliquots of each sample were loaded with a Mo catalyst using an 144 aqueous/methanol (1:1) solution of ammonium dioxydithiomolybdate $\left[\left(\mathrm{NH}_{4}\right)_{2} \mathrm{MoO}_{2} \mathrm{~S}_{2}\right]$.

145 Catalyst weight was $\sim 10 \%$ sample weight for all samples to give a nominal loading of $\sim 1 \%$

146 Mo. Catalyst loaded samples were then lyophilized and weighed aliquots of each sample

147 were loaded into small bespoke borosilicate sample vessels of $7 \mathrm{~mm}$ outer diameter, $1 \mathrm{~mm}$ 148 wall thickness and $10 \mathrm{~mm}$ (small) or $15 \mathrm{~mm}$ (large) overall length. The base of each sample 149 vessel was fitted with a porosity \#1 silica frit to allow gas throughflow (manufactured by 150 Robson Scientific, Sawbridgeworth, UK). Small glass microfiber filters $\sim 5 \mathrm{~mm}$ in diameter 151 were used to line the bottom of the vessel and plug the top of the vessel to keep the sample in 152 place. These are hole-punched using a cork borer from standard Whatman $0.45 \mu \mathrm{m}$ glass 153 microfiber filters.

In order to facilitate higher throughput (i.e. $>1$ sample per reaction) the HyPy reactor setup was modified from that outlined in Ascough et al [12]. In this study, the $1 / 4^{\prime \prime}$ silica trap has been removed and the standard $254 \mathrm{~mm} 9 / 16^{\prime \prime}$ reactor has been replaced with a $475 \mathrm{~mm}$ nipple

157 (see Fig. 1). The upper 196mm of the nipple now functions as the reactor while the bottom $158279 \mathrm{~mm}$ is filled with silica mesh and used as a trap to collect the products of the reaction (i.e. 159 the labile carbon fraction). This larger trap does not require cleaning for upwards of 45 reactions.

Multiple samples (4-9) were loaded one above the other into the HyPy reactor as per the experimental design outlined in Figure 2. After sample loading, the reactor was pressurized with hydrogen to $15 \mathrm{MPa}$ with a flow rate of $5 \mathrm{~L} \mathrm{~min}^{-1}$, then heated using a pre-programmed temperature profile. We used the recommended temperature program previously optimized for PyC quantification where samples are initially heated at a rate of $300^{\circ} \mathrm{C} \min ^{-1}$ to $250^{\circ} \mathrm{C}$, then at a rate of $8^{\circ} \mathrm{C} \min ^{-1}$ until the final hold temperature of $550^{\circ} \mathrm{C}$ for $5 \mathrm{~min}[12,14]$. 


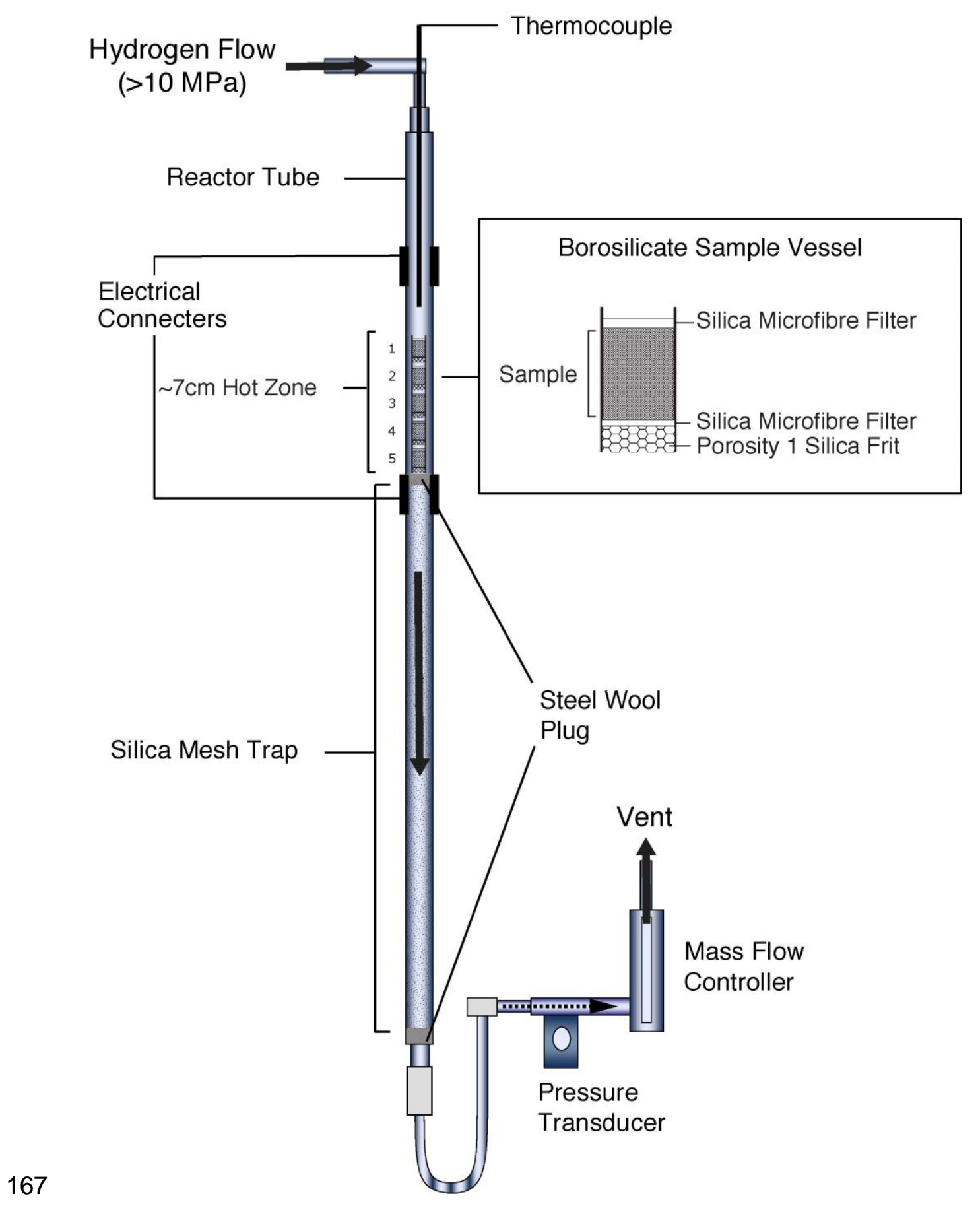

168 Figure 1. Hydropyrolysis reactor schematic showing placement and design of the 169 borosilicate sample vessels, steel wool placeholder, silica trap and direction of Hydrogen 170 flow. 
174 Carbon abundances and isotope compositions of all samples were determined using a 175 Costech Elemental Analyzer (Costech Analytical Technologies Inc., Valencia, CA, 176 USA) fitted with a zero-blank autosampler coupled via a ConFloIV (Thermo Fisher

177 Scientific, Waltham, MA, USA) to a ThermoFinnigan DeltaV ${ }^{\text {PLUS }}$ using Continuous-Flow 178 Isotope Ratio Mass Spectrometry (EA-IRMS) at the Advanced Analytical Unit at James 179 Cook University, Cairns. Stable isotope results are reported as per mil (\%o) deviations from 180 the VPDB reference standard scale for $\delta^{13} \mathrm{C}$ values. Precisions $(2 \sigma)$ on internal standards were 181 better than $\pm 0.2 \%$. Because the catalyst undergoes $(\sim 25 \%)$ weight loss during HyPy, the 182 abundance of residual carbon in the sample after hydrogen pyrolysis is determined as the mass of carbon after treatment relative to the mass of carbon loaded and the results reported as the residual carbon present in the sample $\left(\mathrm{C}_{\mathrm{R}}-\right.$ residual carbon not removable by HyPy). Reproducibility is considered to be $2 \%$ of the value, based on repeated analyses [31].

\subsection{Radiocarbon measurement}

HyPy residues were converted to $\mathrm{CO}_{2}$ by combustion in sealed quartz tubes, and the evolved gas was cryogenically purified and converted to graphite for analysis using Fe/Zn 189 reduction [32]. Sample ${ }^{14} \mathrm{C} /{ }^{13} \mathrm{C}$ ratios were measured by Accelerator Mass Spectrometry [29] 190 at the Scottish Universities Environmental Research Centre. Measured ${ }^{14} \mathrm{C} /{ }^{13} \mathrm{C}$ ratios were normalized to a $\delta^{13} \mathrm{C}$ value of $-25 \%$ and expressed as \% modern carbon (pMC) according to

192 Stuiver and Polach [33].

\subsection{Experimental Design} 195 the materials described in table 1. 
(i) Experiment 1: The purpose of this experiment was to determine the location of the 197 reactor 'hot zone' i.e. the region where the temperature is consistent and hydrogen pyrolysis is effective and reproducible. This zone defines the maximum number of samples that can be run simultaneously using the modified reactor setup. Initially, 9 small (10 mm long) sample vessels containing $\sim 100 \mathrm{mg}$ of a reference material (BCM) were run in quadruplicate. This experiment was repeated with 6 large $(15 \mathrm{~mm}$ long) sample vessels of a composite soil control sample (AGR), also run in quadruplicate.

(ii) Experiment 2: In this experiment, (fig 2a), $\sim 30 \mathrm{mg}$ of anthracite was first used to 204 determine the HyPy ${ }^{14} \mathrm{C}$ background for the radiocarbon experiments. Measurements of anthracite were also performed before and after a HyPy run, to test for any sequential changes in the HyPy instrument ${ }^{14} \mathrm{C}$ background. Two RDC samples were used as controls. In experiment $2 \mathrm{a}, \sim 100 \mathrm{mg}$ (replicate 1 ) or $\sim 50 \mathrm{mg}$ (replicate 2 ) of TBM was placed vertically above three RDC samples in the same reactor. The TIRI standard and three RDC samples were all contained in the same HyPy reactor run to test whether labile carbon is transferred from the sample above to the samples below, in the direction of hydrogen flow, manifest in a

211 measurable increase in radiocarbon in the RDC samples. This experiment was repeated in 212 experiment $2 \mathrm{~b}$, with the addition of a sample vessel filled with $70-200 \mu \mathrm{m}$ silica mesh between the $\sim 50 \mathrm{mg}$ of TBM and RDC samples, replicated twice.

Experiment 3: The purpose of this experiment was to quantify the amount of labile TOC is transferred 'downstream' from one vessel to another over the course of a single reactor run. 216 To do this, 2-10mg of carbon (in the form of Sugarcane Leaves or BCM) was placed in a 217 sample vessel above a series of four silica mesh spacers, with or without an empty sample 218 vessel (acting as a spacer) at position 2. The silica mesh was analysed for $\mathrm{C}$ abundance by 219 Costech elemental analyser immediately following the HyPy. In addition, a background silica 
mesh carbon abundance was determined by successive HyPy runs comprising exclusively silica mesh spacers.

Experiment 4: The purpose of this experiment was to test the degree to which labile 223 carbon released from one sample during a HyPy run, is transferred to another 'downstream' 224 sample within the HyPy reactor. In this experiment an inhouse reference of labile $\mathrm{C}_{3}\left(\mathrm{C}_{4}\right)$ 225 material, $\mathrm{C}_{3}$ rainforest leaves (or $\mathrm{C}_{4}$ sugarcane leaves) was placed in a sample vessel above a vessel loaded with a $\mathrm{C}_{4}\left(\right.$ or $\mathrm{C}_{3}$ ) soil sample containing $\mathrm{PyC}$ of known abundance and stable isotope composition, both with and without a silica mesh spacer at position 2 (fig $2 \mathrm{~d}$ ). This experiment was repeated in quadruplicate.

a) Experiment 1: Position experiment

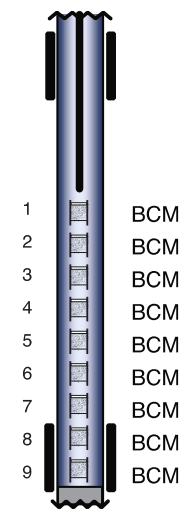

Replicates $=4$

c) Experiment 2b: Radiocarbon Experiment $\mathrm{b}$

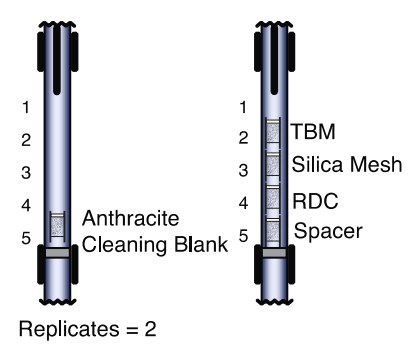

d) Experiment 3: Si Experiment
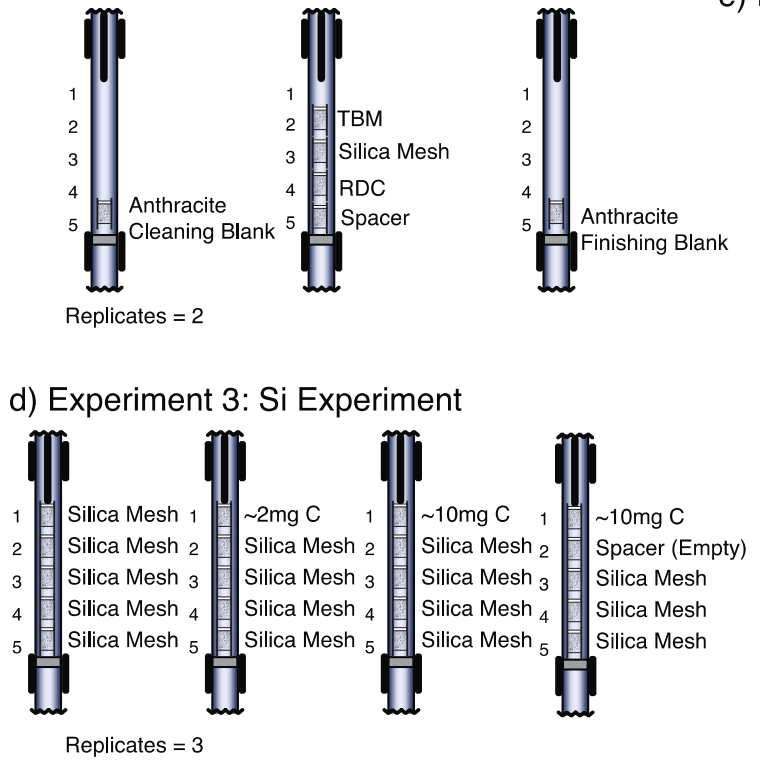

b) Experiment 2a: Radiocarbon Experiment a

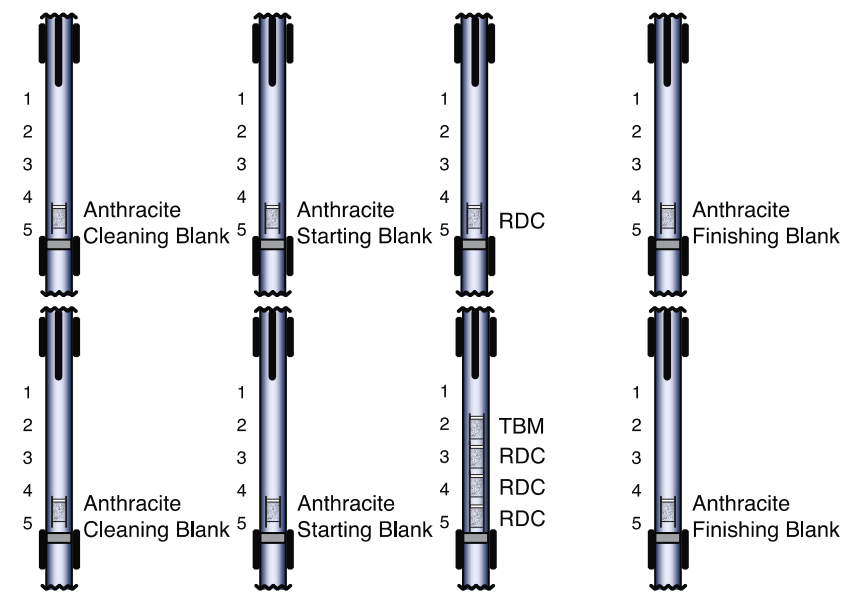

Replicates $=2$ (using $\sim 100 \mathrm{mg}(\sim 50 \mathrm{mg})$ of Tiri Barley Mash)

e) Experiment 4: $\delta^{13} \mathrm{C}$ Experiment
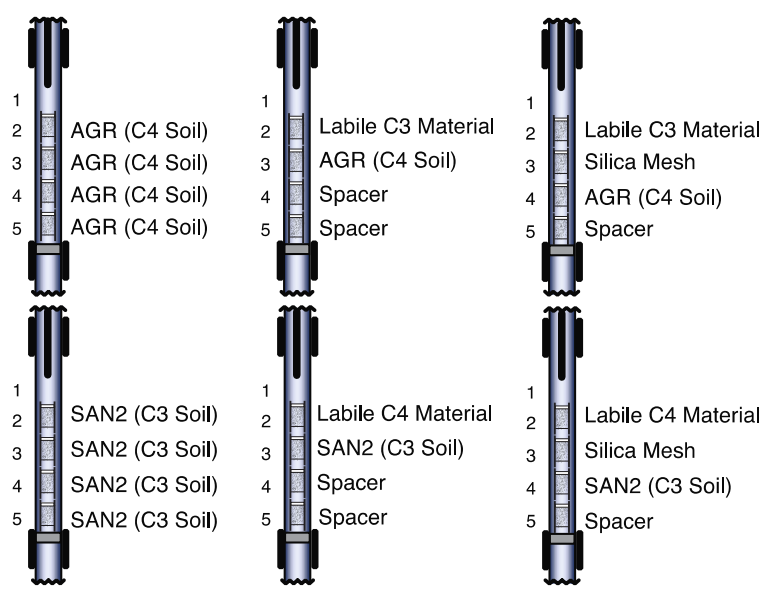

Replicates $=4$ 
230 Figure 2. Experimental design, showing the position and contents of the sample vessels in each HyPy experiment run, the number of replicates and the order of runs. A) position experiment to determine the ideal number of samples per run and the location of the 'hot zone' within the reactor; $B)$ radiocarbon experiment $A$, to determine if running multiple samples simultaneously leads to ${ }^{14} \mathrm{C}$ contamination of samples below; C) radiocarbon experiment $B$, to determine if adding silica mesh spacers between samples reduces ${ }^{14} C$ contamination of the sample below; D) silica mesh experiment to quantify the transfer of labile $C$ down the profile, with or without an empty sample vessel (spacer) E) Replication of the previous experiments to test whether typical $C_{3}\left(C_{4}\right)$ soil samples are contaminated by labile $C_{4}\left(C_{3}\right)$ material from the vessel directly above, and if a silica mesh spacer resolves this.

Pairwise multiple comparison tests were used in experiment 1 to determine whether $\mathrm{PyC} \%$ was influenced by position within the HyPy reactor, and to identify the area within the reactor in which the variability was least (i.e. the 'hot zone') and thus the ideal positioning of the steel wool plug that holds all the vessels in place in the reactor (Figure 1). Pairwise multiple comparison tests were also used in experiment 4 to determine whether the amount of labile carbon above a sample impacts the $\delta^{13} \mathrm{C}$ value of $\mathrm{PyC}$ in a sample downstream.

\section{Results and Discussion}

3.1 Experiment 1: 'hot zone' delimitation

In conventional HyPy, samples are processed individually in a 43 minute HyPy run $251 \quad[12,14]$. To determine the feasibility of running multiple samples simultaneously, multiples of 9 small sample vessels $(10 \mathrm{~mm} ; \mathrm{n}=26$ total $)$ or 5 large sample vessels $(15 \mathrm{~mm} ; \mathrm{n}=20$ total $)$ 
254 determine the maximum number of samples that can be run in tandem without compromising 255 precision. Across all positions PyC ranged from $0.2-1.1 \%(0.1-0.2 \%)$ and $\delta^{13}$ PyC ranged 256 from -26.3 to $-26.6 \%$ ( -15.4 to $-14.2 \%$ ) for the experiments based on BCM (AGR). The 257 summary statistics are given in table 2.

258 Comparison of the results by position in the reactor (fig 3a) shows that results from the $10 \mathrm{~mm}$ 259 vessels (BCM) in position 8 and 9 do not belong to the same population as samples 1-7, 260 indicating that the 'hot zone' is within $\sim 7 \mathrm{~cm}$ of the thermocouple tip in the reactor $(\mu=0.3$ $261 \pm 0.08 \%,-26.5 \pm 0.6 \%$ ), below this reproducibility is diminished. A repeat of the same 262 experiment using $15 \mathrm{~mm}$ vessels (fig 3c: AGR) confirms the $\sim 7 \mathrm{~cm}$ hot zone as there is no 263 statistical difference in $\mathrm{PyC} \%$ between samples in positions $1-5$ i.e. within $\sim 7.5 \mathrm{~cm}$ of the 264 thermocouple $(\mu=0.2 \pm 0.01 \%,-14.7 \pm 0.3 \%$ ). Position affects $\mathrm{PyC} \%$ to a greater extent than $265 \quad \delta^{13} \mathrm{PyC}$ (fig $3 \mathrm{~b}$ and $3 \mathrm{~d}$ ) as there is no statistical difference between any position when using 266 either small or large vessels providing an error $(2 \sigma)$ of $0.6 \%$ and $0.3 \%$ is acceptable. This 267 confirms that multiples of 7 (small $10 \mathrm{~mm}$ vessels) or 5 (large $15 \mathrm{~mm}$ vessels) samples can be 268 accurately run together within a single 45 minute HyPy run, with a precision better than $269 \quad 0.08 \%$ PyC and $0.6 \% \delta^{13} \mathrm{PyC}$, equating to a $400-600 \%$ increase in throughput compared to 270 the conventional 'single sample per run' method. 


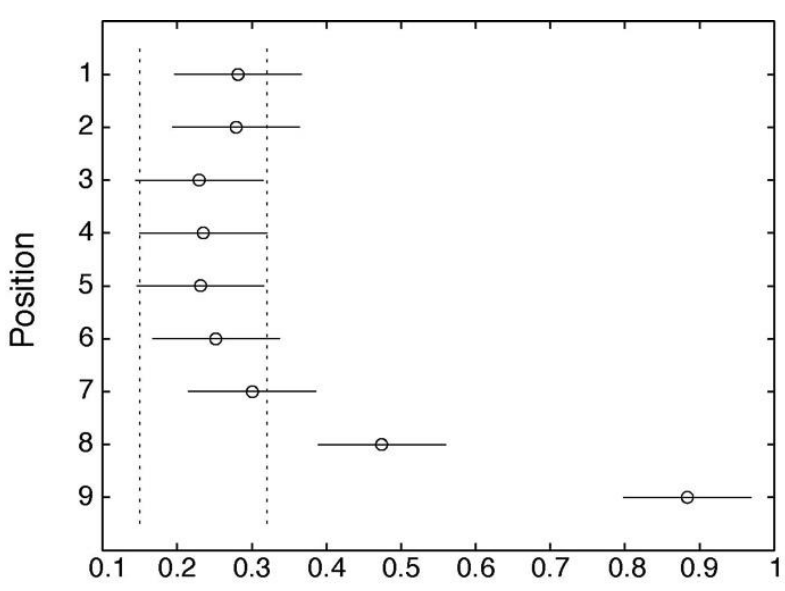

a) $\mathrm{BCM} \mathrm{PyC} \%$

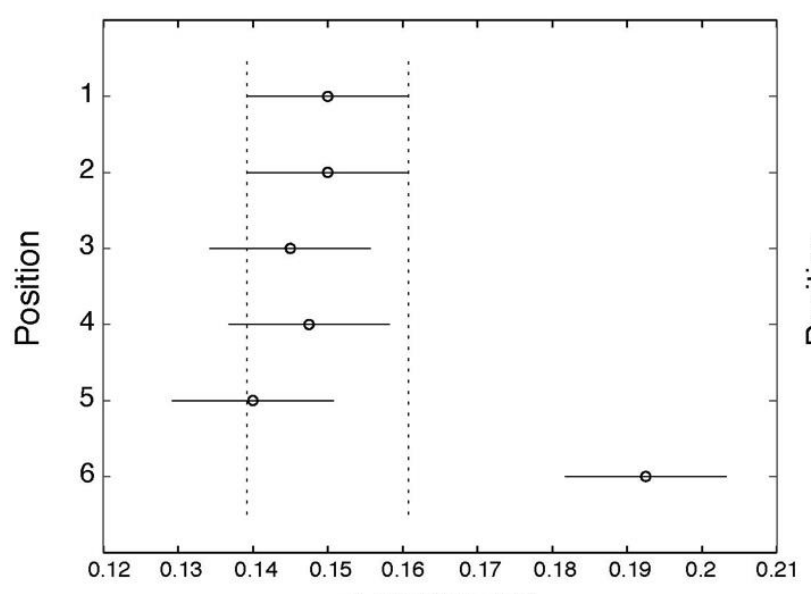

c) AGR PyC $\%$

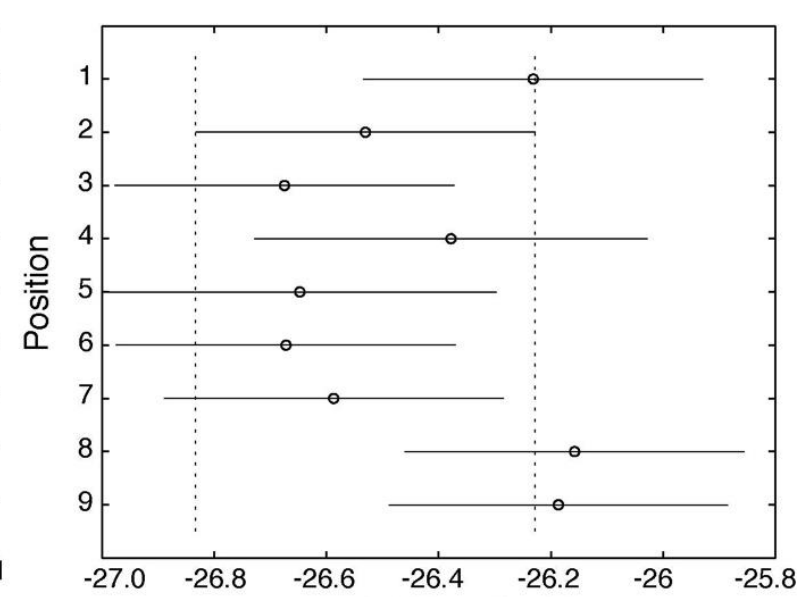

b) $\mathrm{BCM} \delta^{13} \mathrm{PyC}$

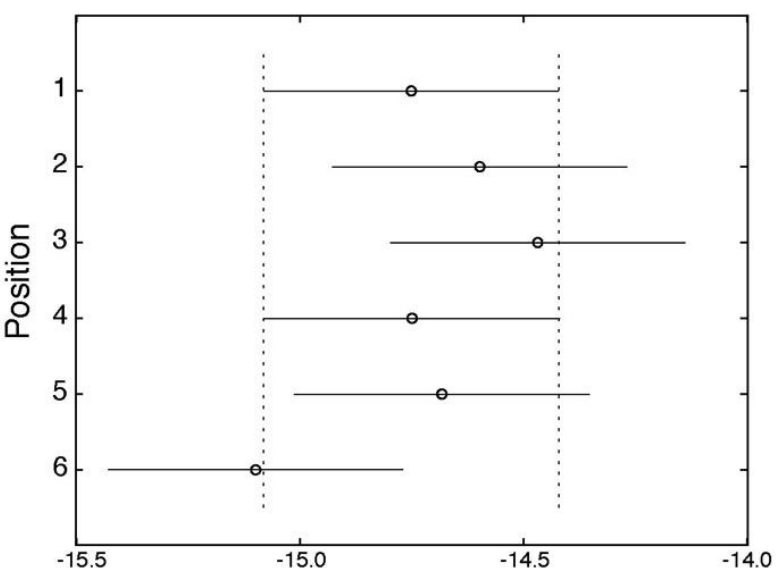

d) AGR $\delta^{13} \mathrm{PyC}$

Figure 3. Pairwise multicomparison plot showing test estimates i.e. group mean $\mu$ (circles), and comparison intervals $\alpha$ (lines) for $10 \mathrm{~mm}$ sample vessels of $B C M P y C \%$ (A) and $\delta^{13} P y C$

274 (B); and 15mm sample vessels of AGR composite soil PyC\% (C) and $\delta^{13} P y C(D)$. and purifying PyC for ${ }^{14} \mathrm{C}$ measurements [12,13,24,25]. Experiment 1 confirmed that it is 278 statistically acceptable to process up to 5 large samples in tandem for PyC quantification by 279 HyPy. In experiment 2, we assess the potential for pre-treating multiple samples simultaneously when radiocarbon measurement of these samples is also required. To determine the appropriate HyPy radiocarbon pretreatment background, a series of anthracite cleaning, and finishing blanks were run in isolation before and after each experiment. 
283 Combining all anthracite runs indicates a HyPy background value of $0.13 \mathrm{pMC}(\mu=0.10,2 \sigma$

$284=0.03, \mathrm{n}=11)$. This is significantly less than the standard internal NERC Radiocarbon

285 Laboratory quartz tube process background value of $0.17 \pm 0.02 \mathrm{pMC}$. The anthracite finishing 286 blanks run immediately after each experiment were indistinguishable from the lab process 287 background $(\mu=0.10, \sigma=0.04)$, meaning there is no detectable additional ${ }^{14} \mathrm{C}$ added by the 288 HyPy process above that of the quartz tube combustion when compared to standard process background values.

These results indicate that normal cleaning between HyPy runs (i.e. manual rinsing of the reactor with dichloromethane, followed by drying of the reactor and complete removal of dichloromethane during the warm-up phase of HyPy treatment, is sufficient to preserve appropriate ${ }^{14} \mathrm{C}$ background values. Indeed, the measured HyPy process background values were consistently lower than the nominal laboratory process background for the quartz tube method of combustion, indicating that the HyPy process may be a particularly 'clean' pretreatment for samples in comparison with other methods applied to PyC (as charcoal), such as the Acid-Base-Acid processing protocol. This result is promising and warrants further investigation.

3.3 Experiment 2b: Assessment of downstream contamination potential by radiocarbon

To assess the potential for transfer of exogenous ${ }^{14} \mathrm{C}$ between samples downstream in the reactor, labile TBM (116.35 pMC) was placed above three samples of RDC. This represents a 'worst case' scenario, where radiocarbon dead material is contaminated with modern carbon, and where the source of the modern contamination is completely labile in the reactor. RDC samples positioned directly below the TBM returned 0.477 and $0.429 \pm 0.02 \mathrm{pMC}$ 305 respectively (equivalent to $42938 \pm 150$ and $43801 \pm 167{ }^{14} \mathrm{C}$ years, Fig 4 ) in duplicate runs.

306 The radiocarbon content of the RDC samples reduces further down the profile in the reactor 
307 to $\leq 0.189 \pm 0.01\left(\leq 50398 \pm 252{ }^{14} \mathrm{C}\right.$ years $)$ which is within error of the quartz tube 308 background (at position 4 in Fig 2b) and below background levels after that.

309 The addition of a silica mesh spacer between the TBM and RDC samples (see fig 2c for 310 placement), reduces the transfer of modern carbon downstream to levels that are not 311 distinguishable from the process background. These results should be interpreted as "worst 312 case' given the very high loading of 100\% labile (under HyPy conditions) and 'modern' (in

$313{ }^{14} \mathrm{C}$ terms) carbon that was used as the 'contaminant' in this case. Positioning a radiocarbon314 dead sample below a significant amount of modern contaminant ( 20-45mg of modern 315 carbon), at worst, returns a value of $<0.5 \mathrm{pMC}$ however the use of a silica mesh spacer 316 between samples is sufficient to ensure that no subsequent samples are affected. Therefore, 317 even when pretreating 'worst case' samples for radiocarbon analysis via HyPy (i.e. high 318 levels of modern labile $\mathrm{C}$ that has a significantly different ${ }^{14} \mathrm{C}$ age to other material analysed 319 in the same run), these results indicate that it is acceptable to process up to 3 samples 320 simultaneously in a 45 minute HyPy run when interspersed with silica mesh spacers. 


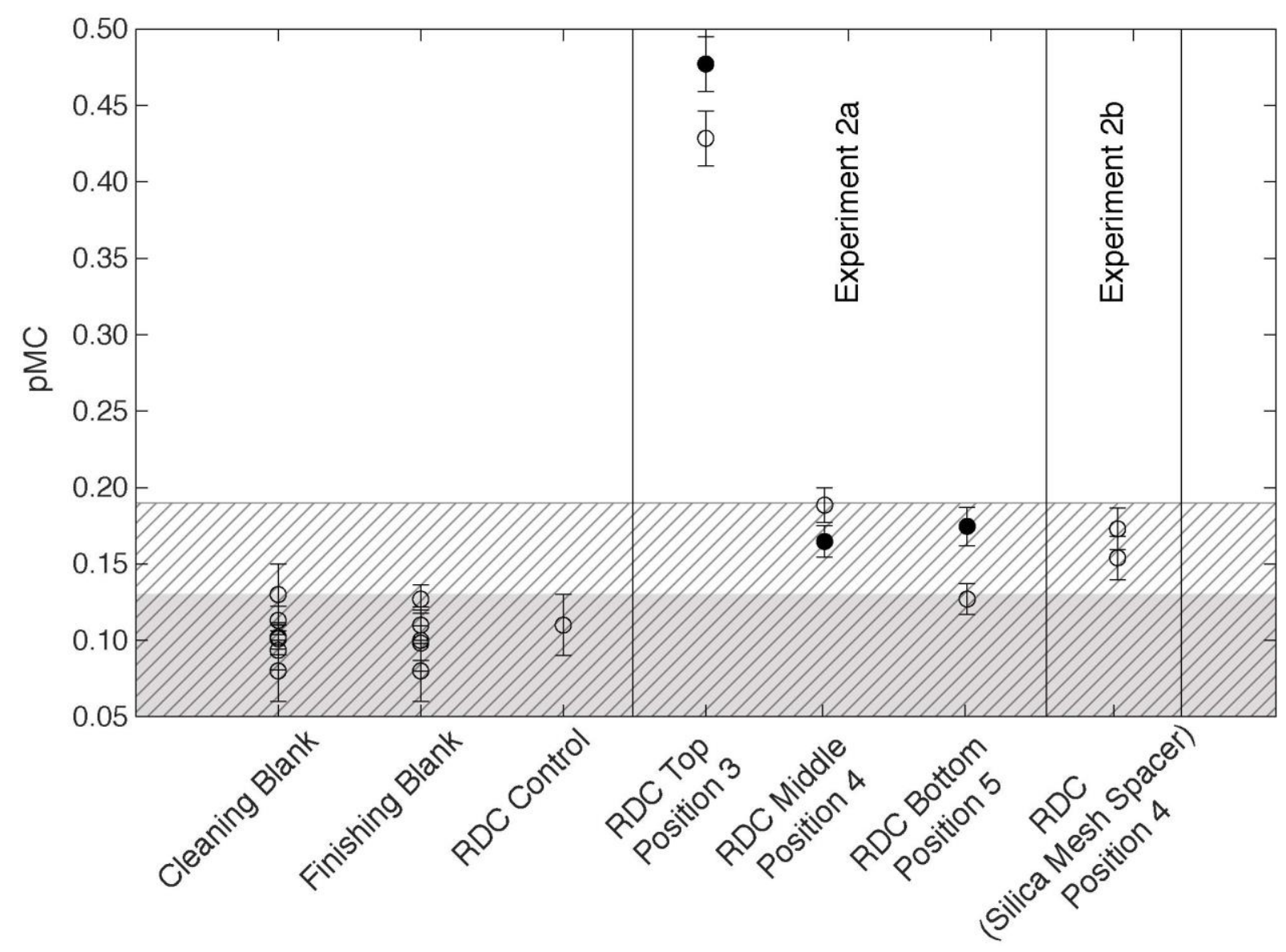

322

323 Figure 4. Radiocarbon results in pMC (without background correction) for experiment 2

indicating $2 \sigma$ error. Dashed shading indicates the accepted NERC Radiocarbon laboratory internal quartz tube process background value of $0.17 \pm 0.02$ pMC, grey shading indicates the 'HyPy' process background value of $0.10 \pm 0.06$ pMC for comparison. RDC top, middle and bottom indicates the location of the radiocarbon dead charcoal below the barley mash vessel 328 (116.35 pMC), no silica mesh spacer was used for these samples, filled (unfilled) circles distinguish the experiments using 100mg ( 50mg) of barley mash.

3303.4 Experiment 3: Assessment of downstream contamination potential by labile C

To quantify the amount of labile carbon transferred downstream to vessels lower in the reactor column, a vessel containing labile carbon was placed in position 1 atop silica mesh spacers positioned downstream (refer to fig $2 \mathrm{~d}$ for placement). The silica mesh was analysed 334 for $\mathrm{C}$ abundance immediately following the HyPy run (see Fig 5). The silica mesh 
335 background (blank) was determined to be $0.005 \pm 0.004 \mathrm{mg}$ carbon. The high TOC 336 experiment resulted in a transfer of $0.07 \pm 0.02 \mathrm{mg}$ carbon $(0.67 \%$ of total labile carbon) from

337 the high organic carbon sample in position 1 onto the silica mesh directly below (at position

338 2). The addition of an empty vessel (empty spacer) at position 2 between the high TOC 339 sample and the silica mesh below (at position 3), reduced the transfer to $0.02 \pm 0.02 \mathrm{mg}$ $340(0.09 \%$ of total labile carbon), which is an $86.6 \%$ reduction in the amount of $\mathrm{C}$ transfer from 341 the sample above and within $\sim 10 \%$ of the sample in the same position (position 3 ) in the 342 previous high TOC experiment.

343 All silica mesh samples in the low TOC experiment are below the measured background 344 (effectively 0\% carbon). Silica mesh in positions 3, 4 and 5 in the high TOC experiment and 345 positions 4 and 5 in the spacer experiment are also below background (effectively $0 \%$ 346 carbon). In practice, it is unlikely that a 'typical' sample in a large vessel could accommodate $347>10 \mathrm{mg}$ of carbon. In this worst case scenario, there is a limited effect on the sample 348 immediately beneath an organic-rich sample and little to no effect on the remaining samples 349 downstream. This effect is small (i.e. $<0.73 \%$ carbon is transferred from the sample above to 350 the sample below) and can easily be addressed with the interposition of an empty spacer (or 351 silica mesh spacer). 


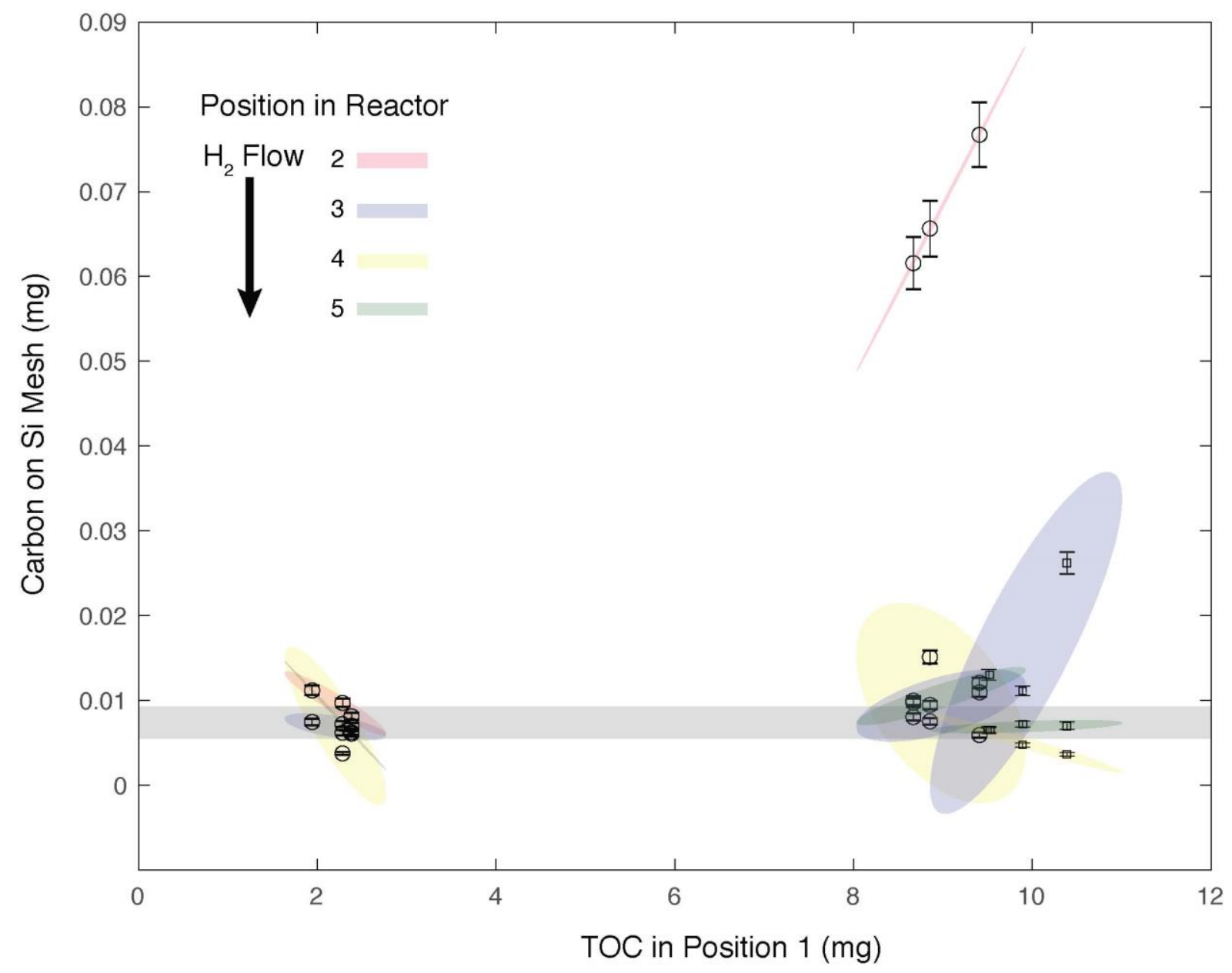

353 Figure 5. Milligrams of carbon transferred onto the silica mesh samples positioned below a 354 low TOC organic (BCM) or a high TOC organic (Sugarcane Leaves) (open circles) and a high TOC organic material with an empty spacer at position 2 acting as a spacer (open squares). Ellipses indicate the 95\% confidence interval at each position (coloured shading).

357 Grey shading indicates the background silica mesh blank value of $0.005 \pm 0.004 \mathrm{mg}(2 \sigma)$.

$358 \quad 3.5$ Experiment 4: Assessment of downstream contamination potential by $\delta^{13} \mathrm{C}$

359 Isotopically dissimilar labile carbon-rich material was placed in position 1 to test whether 360 the $\delta^{13} \mathrm{C}$ value of a sample below (in position 2) is affected. The $\mathrm{C} 4(\mathrm{C} 3)$ soils below the $361 \mathrm{C} 3(\mathrm{C} 4)$ organics in position 1 , returned $\delta^{13} \mathrm{C}$ values which were significantly different from 362 the control ( $p$ value $=0.01$ and 0.03 respectively), lowering (increasing) the $\delta^{13} \mathrm{PyC}$ of the 363 material below relative to the $\mathrm{C} 4(\mathrm{C} 3)$ control (see fig 6). This effect is minimal in samples 
with higher $\mathrm{PyC} \%$ (fig 6b), and slightly more noticeable in samples with less $\mathrm{PyC} \%$ (fig 6a) resulting in an offset of $0.3 \%$ and $0.6 \%$; and $0.06 \%$ and $0.01 \%$. The addition of an amorphous silica mesh spacer between the two materials negates any measurable transfer of labile carbon from the organic material above onto the soil sample below $(\mathrm{t}=-1.33$ and 0.86 respectively). In short, HyPy treatment of multiple isotopically divergent materials ( $>10 \%$ in this case) can offset $\delta^{13} \mathrm{PyC}$ in downstream samples by as much as $0.6 \%$ however, this can be negated by the use of a silica mesh spacer.

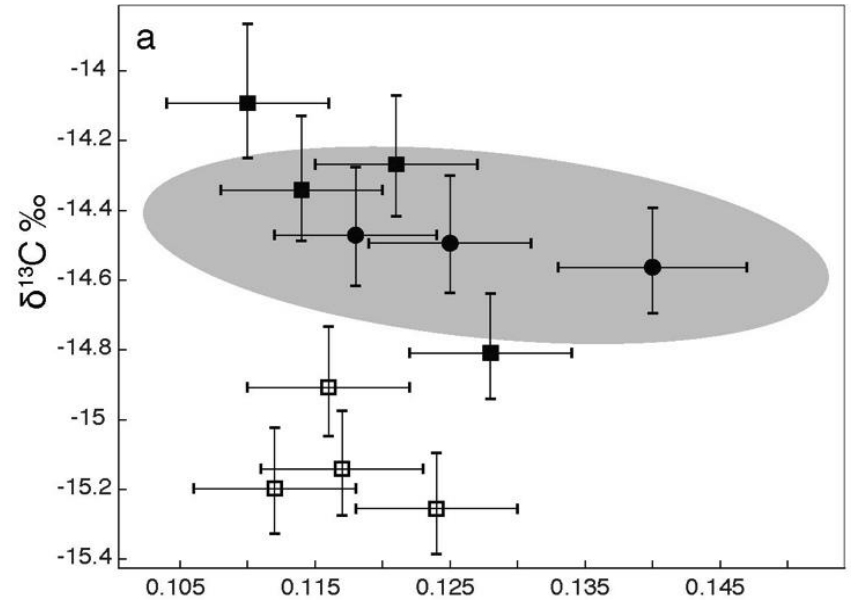

Pyrogenic Carbon (\%)

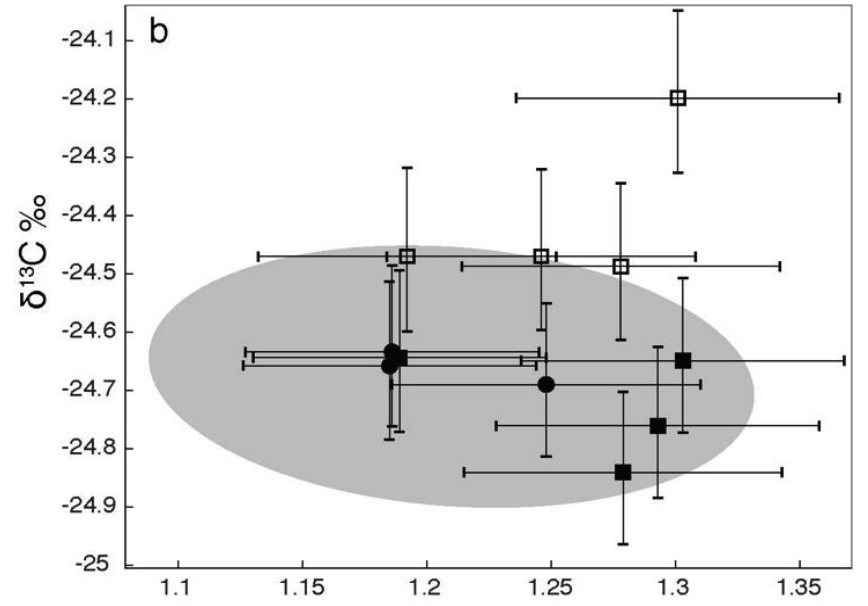

Pyrogenic Carbon (\%)

372 Figure 6. $\delta^{13} \mathrm{PyC}$ and $\mathrm{PyC}(\%)$ of $\mathrm{C} 4(\mathrm{C} 3)$ soil samples in sample vessels positioned below C3(C4) organics $a(b)$ in position 1. Black circles denote the C4(C3) controls, black squares indicate that a silica mesh spacer was placed between the organic and soil sample vessels, open squares indicate that a silica mesh spacer was not used. Grey shading indicates the 95\% confidence interval of the control samples $C 4$ soil (A) and $C 3$ soil (B) respectively, $\delta^{13} \mathrm{PyC}$ and $\mathrm{PyC}(\%)$ error determined as per Wurster et al [31].

\section{Conclusions}

HyPy has previously been shown to produce accurate and precise determinations of radiocarbon abundance [12,13] and $\delta^{13} \mathrm{C}$ value of a well defined component of PyC $[31,6]$. 
are identical and thus multiples of 7 (small) or 5 (large) samples, in bespoke borosilicate vessels, can be run in tandem within a single 45 minute HyPy run, with a precision $(2 \sigma)$ better than $0.08 \%$ PyC and $0.6 \%$ o $\delta^{13}$ PyC. Experiments using labile carbon-rich samples immediately above samples of highly divergent isotope composition were able to detect trace cross-contamination. However, radiocarbon dead PyC positioned immediately below 20$45 \mathrm{mg}$ of labile carbon with a high radiocarbon content resulted in $<0.5 \mathrm{pMC}$ transfer into the radiocarbon dead sample immediately below. Similarly, small transfers were found using samples widely divergent in $\delta^{13} \mathrm{C}$ value. Cross-contamination only occurred in the sample immediately below the labile carbon source and did not carry downstream to samples lower in the reactor. In all cases, the use of a silica mesh spacer eliminated cross-contamination between samples. Therefore, even when pretreating 'dirty' samples for radiocarbon or stable isotope analysis using HyPy, the results indicate that it is acceptable to process up to 3 samples simultaneously. In addition, lower radiocarbon backgrounds were obtained using the HyPy process in comparison to standard pretreatment protocols at NCRF, suggesting further potential for application as a low blank pretreatment for radiocarbon dating should be further investigated.

The step that currently limits the application of hydrogen pyrolysis for PyC isolation and 399 analysis is the 43 minute temperature ramp to remove labile carbon (and subsequent 23 minute period required for cooling), with only $\sim 7$ samples able to be processed in a single day. This study suggests that 3 (with spacers) to 7 (without spacers) samples can be processed 402 in a single run without diminishing accuracy or precision, enabling $20-50$ samples to be 403 processed in a day. This is comparable to the number of samples that can be run by EA-IRMS 404 for stable isotopes in a day, removing the current bottleneck in routine application to larger 405 scale projects where quantification of $\mathrm{PyC}$ and determination of isotope composition is required. 


\section{References}

4121 Bird MI, Wynn JG, Saiz G, Wurster CM, McBeath A. The Pyrogenic Carbon Cycle. Annual Review of Earth and Planetary Sciences. 2015;43(1):273-298. doi:10.1146/annurev-earth-060614-105038.

2 Santin C, Doerr SH, Kane ES, Masiello CA, Ohlson M, de la Rosa JM, Preston CM, Dittmar T. Towards a global assessment of pyrogenic carbon from vegetation fires. Glob Chang Biol. 2016;22(1):76-91. doi:10.1111/gcb.12985.

3 Reisser M, Purves RS, Schmidt MWI, Abiven S. Pyrogenic Carbon in Soils: A Literature-Based Inventory and a Global Estimation of Its Content in Soil Organic Carbon and Stocks. Frontiers in Earth Science. 2016;4doi:10.3389/feart.2016.00080.

425

426

427

428

429

430

431

432

433

434

435

436

437

438

439

440

441

442

443

444

445

446

447

Koele N, Bird M, Haig J, Marimon BH, Marimon BS, Phillips OL, de Oliveira EA, Quesada CA, Feldpausch TR. Amazon Basin forest pyrogenic carbon stocks: First estimate of deep storage. Geoderma. 2017;306:237-243. doi:10.1016/j.geoderma.2017.07.029.

5 Sohi SP, Krull E, Lopez-Capel E, Bol R. Chapter 2 - A Review of Biochar and Its Use and Function in Soil. In: Sparks, D. L. ed. Advances in Agronomy. Elsevier; 2010:4782.

6 Wurster CM, McBeath AV, Bird MI. The carbon isotope composition of semi-labile and stable pyrogenic carbon in a thermosequence of $\mathrm{C} 3$ and $\mathrm{C} 4$ derived char. Organic Geochemistry. 2015;81:20-26. doi:10.1016/j.orggeochem.2015.01.008.

7 Hammes K, Schmidt MWI, Smernik RJ, Currie LA, Ball WP, Nguyen TH, Louchouarn P, Houel S, Gustafsson O, Elmquist M, Cornelissen G, Skjemstad JO, Masiello CA, Song J, Peng P, Mitra S, Dunn JC, Hatcher PG, Hockaday WC, Smith DM, Hartkopf-Froeder C, Boehmer A, Luer B, Huebert BJ, Amelung W, Brodowski S, Huang L, Zhang W, Gschwend PM, Flores-Cervantes DX, Largeau C, Rouzaud JN, Rumpel C, Guggenberger G, Kaiser K, Rodionov A, Gonzalez-Vila FJ, GonzalezPerez JA, de la Rosa JM, Manning DAC, Lopez-Capel E, Ding L. Comparison of quantification methods to measure fire-derived (black/elemental) carbon in soils and sediments using reference materials from soil, water, sediment and the atmosphere. Global Biogeochemical Cycles. 2007;21(3)doi:10.1029/2006gb002914. $15 \mathrm{~N}-\mathrm{NMR}$ spectroscopic examination of the transformation of organic nitrogen in 
plant biomass during thermal treatment. Soil Biology and Biochemistry. 1996;28(8):1053-1060. doi:10.1016/0038-0717(96)00078-8.

9 Chang Z, Tian L, Li F, Zhou Y, Wu M, Steinberg CEW, Dong X, Pan B, Xing B. Benzene polycarboxylic acid - A useful marker for condensed organic matter, but not for only pyrogenic black carbon. Sci Total Environ. 2018;626:660-667. doi:10.1016/j.scitotenv.2018.01.145.

10 Love GD, Snape CE, Carr AD, Houghton RC. Release of covalently-bound alkane biomarkers in high yields from kerogen via catalytic hydropyrolysis. Organic Geochemistry. 1995;23(10):981-986. doi:10.1016/0146-6380(95)00075-5.

11 Craig OE, Love GD, Isaksson S, Taylor G, Snape CE. Stable carbon isotopic characterisation of free and bound lipid constituents of archaeological ceramic vessels released by solvent extraction, alkaline hydrolysis and catalytic hydropyrolysis. Journal of Analytical and Applied Pyrolysis. 2004;71(2):613-634. doi:10.1016/j.jaap.2003.09.001.

12 Ascough PL, Bird MI, Brock F, Higham TFG, Meredith W, Snape CE, Vane CH. Hydropyrolysis as a new tool for radiocarbon pre-treatment and the quantification of black carbon. Quaternary Geochronology. 2009;4(2):140-147. doi:10.1016/j.quageo.2008.11.001.

13 Ascough PL, Bird MI, Meredith W, Wood RE, Snape CE, Brock F, Higham TFG, Large DJ, Apperley DC. Hydropyrolysis: Implications for Radiocarbon Pretreatment and Characterization of Black Carbon. Radiocarbon. 2010;52(3):1336-1350. doi:10.1017/S0033822200046427.

14 Meredith W, Ascough PL, Bird MI, Large DJ, Snape CE, Sun Y, Tilston EL. Assessment of hydropyrolysis as a method for the quantification of black carbon using standard reference materials. Geochimica Et Cosmochimica Acta. 2012;97:131147. doi:10.1016/j.gca.2012.08.037.

15 Robert AM, Grotheer H, Greenwood PF, McCuaig TC, Bourdet J, Grice K. The hydropyrolysis (HyPy) release of hydrocarbon products from a high maturity kerogen associated with an orogenic Au deposit and their relationship to the mineral matrix. Chemical Geology. 2016;425:127-144. doi:10.1016/j.chemgeo.2016.01.028.

16 Rombola AG, Fabbri D, Meredith W, Snape CE, Dieguez-Alonso A. Molecular characterization of the thermally labile fraction of biochar by hydropyrolysis and pyrolysis-GC/MS. Journal of Analytical and Applied Pyrolysis. 2016;121:230-239. doi:10.1016/j.jaap.2016.08.003.

17 Sephton MA, Love GD, Meredith W, Snape CE, Sun CG, Watson JS. Hydropyrolysis: A new technique for the analysis of macromolecular material in meteorites. Planetary and Space Science. 2005;53(12):1280-1286. doi:10.1016/j.pss.2005.06.008. 
18 Foereid B, Lehmann J, Wurster C, Bird M. Presence of Black Carbon in Soil due to Forest Fire in the New Jersey Pine Barrens. Journal of Earth Science and Engineering. 2015;5(2):91-97. doi:10.17265/2159-581x/2015.02.001.

19 Santin C, Doerr SH, Merino A, Bucheli TD, Bryant R, Ascough P, Gao X, Masiello CA. Carbon sequestration potential and physicochemical properties differ between wildfire charcoals and slow-pyrolysis biochars. Sci Rep. 2017;7(1):11233. doi:10.1038/s41598-017-10455-2.

20 Wurster CM, Saiz G, Schneider MPW, Schmidt MWI, Bird MI. Quantifying pyrogenic carbon from thermosequences of wood and grass using hydrogen pyrolysis. Organic Geochemistry. 2013;62:28-32. doi:10.1016/j.orggeochem.2013.06.009.

510

511

512

513

514

21 Meredith W, McBeath A, Ascough P, Bird MI. Analysis of biochars by hydropyrolysis (HyPy). In: Singh, B., Camps Arbestain, M. \& Lehmann, J. eds. Biochar: A Guide to Analytical Methods. Clayton: CRC Press; 2017.

22 Grotheer H, Greenwood PF, McCulloch MT, Böttcher ME, Grice K. $\delta 34 \mathrm{~S}$ character of organosulfur compounds in kerogen and bitumen fractions of sedimentary rocks. Organic Geochemistry. 2017;110:60-64. doi:10.1016/j.orggeochem.2017.04.005.

23 Saiz G, Goodrick I, Wurster C, Nelson PN, Wynn J, Bird M. Preferential Production and Transport of Grass-Derived Pyrogenic Carbon in NE-Australian Savanna Ecosystems. Frontiers in Earth Science. 2018;5(1):115. doi:10.3389/feart.2017.00115.

24 Field E, Marx S, Haig J, May JH, Jacobsen G, Zawadzki A, Child D, Heijnis H, Hotchkis M, McGowan H, Moss P. Untangling geochronological complexity in organic spring deposits using multiple dating methods. Quaternary Geochronology. 2018;43:50-71. doi:10.1016/j.quageo.2017.10.002.

25 Zhang XY, Huang DS, Deng H, Snape C, Meredith W, Zhao Y, Du Y, Chen X, Sun YG. Radiocarbon dating of charcoal from the Bianjiashan site in Hangzhou: New evidence for the lower age limit of the Liangzhu Culture. Quaternary Geochronology. 2015;30:9-17. doi:10.1016/j.quageo.2015.07.001.

26 Llorente M, Turrion MB, Glaser B. Rapid and economical quantification of black carbon in soils using a modified benzene polycarboxylic acids (BPCA) method. Organic Geochemistry. 2018;115:197-204. doi:10.1016/j.orggeochem.2017.11.005.

27 Gulliksen S, Scott M. Report of the TIRI workshop, Saturday 13 August 1994. Radiocarbon. 1995;37(2):820-821. doi:10.1017/S0033822200031404.

28 Rozanski K, Stichler W, Gonfiantini R, Scott EM, Beukens RP, Kromer B, Van Der Plicht J. The IAEA 14C Intercomparison Exercise 1990. Radiocarbon. 2016;34(3):506-519. doi:10.1017/s0033822200063761.

29 Bird MI, Levchenko V, Ascough PL, Meredith W, Wurster CM, Williams A, Tilston EL, Snape CE, Apperley DC. The efficiency of charcoal decontamination for 
radiocarbon dating by three pre-treatments - ABOX, ABA and hypy. Quaternary Geochronology. 2014;22:25-32. doi:10.1016/j.quageo.2014.02.003.

30 Love GD, McAulay A, Snape CE, Bishop AN. Effect of process variables in catalytic hydropyrolysis on the release of covalently bound aliphatic hydrocarbons from sedimentary organic matter. Energy \& Fuels. 1997;11(3):522-531. doi:10.1021/ef960194x.

31 Wurster CM, Lloyd J, Goodrick I, Saiz G, Bird MI. Quantifying the abundance and

32 Slota PJ, Jull AJT, Linick TW, Toolin LJ. Preparation of Small Samples for 14C

33 Stuiver M, Polach HA. Discussion Reporting of 14C Data. Radiocarbon.

33 Stuiver M, Polach HA. Discussion Reporting of
2016;19(3):355-363. doi:10.1017/s0033822200003672.

Accelerator Targets by Catalytic Reduction of CO. Radiocarbon. 1987;29(2):303306. doi:10.1017/s0033822200056988. stable isotope composition of pyrogenic carbon using hydrogen pyrolysis. Rapid Commun Mass Spectrom. 2012;26(23):2690-2696. doi:10.1002/rcm.6397.

(1)


Table 1. Consensus values for in-house materials used in experiments to assess variation in measured PyC abundance and $\delta^{13} C$ value when combining multiple samples during a single hydropyrolysis run (error is reported as $2 \sigma$ ).

\begin{tabular}{|l|c|c|c|c|c|}
\hline \multicolumn{2}{|c|}{ Soil Samples } & TOC (\%) & $\delta^{13}$ C & PyC (\%) & $\delta^{13} \mathrm{PyC}$ \\
\hline & BC Mollisol (BCM) & $2.04 \pm 0.02$ & $-25.57 \pm 0.2$ & $0.25 \pm 0.1$ & $-26.47 \pm 0.6$ \\
\hline C3 & SAN2 Surface & $13.44 \pm 0.6$ & $-25.73 \pm 0.5$ & $1.18 \pm 0.1$ & $-24.66 \pm 0.2$ \\
\hline C4 & AGR & $1.51 \pm 0.1$ & $-16.51 \pm 0.1$ & $0.15 \pm 0.01$ & $-14.74 \pm 0.3$ \\
\hline C3 & Rainforest Leaves & $44.08 \pm 4.4$ & $-33.47 \pm 0.1$ & N/A & N/A \\
\hline C4 & Sugarcane Leaves & $42.11 \pm 4.2$ & $-11.86 \pm 0.3$ & N/A & N/A \\
\hline
\end{tabular}

583

584

585

586

587

588

589

590

591

592

593 
Table 2. Position experiment summary statistics

\begin{tabular}{|c|c|c|c|c|c|}
\hline \multirow[b]{2}{*}{$\mathrm{BCM}$} & \multirow[b]{2}{*}{ Position } & \multicolumn{2}{|c|}{$\mathrm{PyC}(\%)$} & \multicolumn{2}{|c|}{$\delta^{13} \mathrm{PyC}(\%)$} \\
\hline & & $\mu$ & $2 \sigma$ & $\mu$ & $2 \sigma$ \\
\hline & 1 & 0.3 & 0.04 & -26.2 & 0.3 \\
\hline & 2 & 0.3 & 0.12 & -26.2 & 0.4 \\
\hline & 3 & 0.2 & 0.02 & -26.6 & 0.4 \\
\hline & 4 & 0.2 & 0.04 & -26.7 & 0.3 \\
\hline & 5 & 0.2 & 0.04 & -26.7 & 0.2 \\
\hline & 6 & 0.2 & 0.08 & -26.4 & 0.9 \\
\hline & 7 & 0.3 & 0.08 & -26.7 & 0.2 \\
\hline & 8 & 0.5 & 0.18 & -26.5 & 0.3 \\
\hline & 9 & 0.9 & 0.36 & -26.2 & 1 \\
\hline \multirow[t]{6}{*}{ AGR } & 1 & 0.2 & 0.01 & -14.8 & 0.4 \\
\hline & 2 & 0.2 & 0.01 & -14.7 & 0.2 \\
\hline & 3 & 0.1 & 0.03 & -14.6 & 0.5 \\
\hline & 4 & 0.2 & 0.04 & -14.7 & 0.4 \\
\hline & 5 & 0.1 & 0.02 & -14.9 & 0.1 \\
\hline & 6 & 0.2 & 0.07 & -15.3 & 0.8 \\
\hline
\end{tabular}

595 